\title{
Clinical, histological and therapeutical aspects in the management of uterine and extrauterine stromal sarcomas: Case reports
}

\author{
LAURA FLORENTINA REBEGEA ${ }^{1-3}$, DOREL FIRESCU ${ }^{4,5}$, RODICA MARCELA ANGHEL ${ }^{6,7}$, \\ LAURENTIA GALES $^{6,7 *}$, ANA MARIA ILIE ${ }^{1}$, MIHAELA EMILIA DUMITRU $^{1}$, \\ MIHAELA CRAESCU ${ }^{1,8}$, ELENA NICULET $^{8^{*}}$, ALIN LAURENTIU TATU $^{2,3,9}$, \\ MARIANA STUPARU CRETU ${ }^{3,10}$, MIHAELA LUNGU ${ }^{2,11}$ and ANCA IULIA NEAGU ${ }^{8}$ \\ ${ }^{1}$ Department of Radiotherapy, 'Sfantul Apostol Andrei' Emergency Clinical Hospital, 800578 Galati; \\ ${ }^{2}$ Medical Clinical Department, Faculty of Medicine and Pharmacy, 'Dunarea de Jos' University; \\ ${ }^{3}$ Research Center in The Field of Medical and Pharmaceutical Sciences, ReFORM-UDJ, 'Dunarea de Jos' University, \\ 800010 Galati; ${ }^{4}$ IInd Clinic of Surgery, 'Sfantul Apostol Andrei' Emergency Clinical Hospital of Galati, 800578 Galati; \\ ${ }^{5}$ Surgical Clinical Department, Faculty of Medicine and Pharmacy, 'Dunarea de Jos' University, 800010 Galati; \\ ${ }^{6}$ Radiology, Oncology and Hematology Department, 'Carol Davila' University of Medicine and Pharmacy, \\ 050474 Bucharest; ${ }^{7}$ Clinical Department of Radiotherapy II, 'Prof. Dr. Alexandru Trestioreanu' Oncology Institute, \\ 022328 Bucharest; ${ }^{8}$ Department of Morphological and Functional Sciences, Faculty of Medicine and Pharmacy, \\ 'Dunarea de Jos' University, 800010 Galati; 'Dermatology Department, 'Sfanta Cuvioasa Parascheva' \\ Clinical Hospital of Infectious Diseases, 800179 Galati; ${ }^{10}$ Family Planning Department, 'Buna Vestire' \\ Clinical Hospital of Obstetrics and Gynecology, 800151 Galati; ${ }^{11}$ Department of Neurology, \\ 'Sfantul Apostol Andrei' Emergency Clinical Hospital of Galati, 800578 Galati, Romania
}

Received August 6, 2021; Accepted September 7, 2021

DOI: $10.3892 /$ etm.2021.10891

\begin{abstract}
Correspondence to: Dr Mariana Stuparu Cretu, Research Center in The Field of Medical and Pharmaceutical Sciences, ReFORM-UDJ, 'Dunarea de Jos' University, 35 Al. I. Cuza Street, 800010 Galati, Romania

E-mail: marianascretu@yahoo.com

Dr Mihaela Lungu, Department of Neurology, 'Sfantul Apostol Andrei' Emergency Clinical Hospital of Galati, 177 Brailei Street, 800578 Galati, Romania

E-mail: mihaelalungu17@yahoo.com

"Contributed equally
\end{abstract}

Abbreviations: ESS, endometrial stromal sarcoma; EESS, extrauterine endometrial stromal sarcoma; LG-ESS, low-grade endometrial stromal sarcoma; HG-ESS, high-grade endometrial stromal sarcoma; EBRT, external radiotherapy; 2D, two-dimensional; 3DCRT, three-dimensional conformational radiotherapy; IHC, immunohistochemistry; CT, computer tomography; H\&E, hematoxylin and eosin; HHF35, anti-muscle actin antibody; MRI, magnetic resonance imaging; DFS, disease-free survival; PFFS, pelvic failure-free survival; LRFFS, local-regional failure-free survival; HPF, high-power field; HPE, histopathological examination; GIST, gastrointestinal stromal tumor; PEComa, perivascular epithelioid cell tumor

Key words: endometrial stromal sarcoma, surgery, radiotherapy, immunohistochemistry, extrauterine
Abstract. Endometrial stromal sarcoma (ESS) is a rare tumor, predominantly occurring as a primary tumor of the uterus. Rare cases of primary extrauterine ESS (EESS) have been reported. Low-grade ESS (LG-ESS) is more common than high-grade ESS (HG-ESS). We present five cases of ESS and one case of EESS. All cases received external radiotherapy (EBRT) at the Radiotherapy Department of the Emergency Clinical Hospital 'Sfantul Apostol Andrei' Galati, during 2004-2020. Five cases underwent EBRT in two-dimensional (2D) technique and only one patient received EBRT with three-dimensinal conformational radiotherapy (3DCRT) technique with a linear accelerator, Elekta Synergy. Five patients were referred to postoperative radiotherapy after hysterectomy. The median age of the patients was 57.4 years. One patient was referred to radiotherapy with palliative intent. EESS localized in the retroperitoneum, in the para-aortic region, was identified in one 64-year-old patient with a personal history of hysterectomy and bilateral salpingo-oophorectomy in 1997; EESS was complicated with vertebral extension at the L1-L2 level and spinal cord compression syndrome. ESS represents a rare diagnosis and a high- or low-grade tumor profile is distinguished by immunohistochemistry (IHC) tests. Up to $30 \%$ of patients have EESS at presentation. The treatment of ESS is multimodal, its management requiring a multidisciplinary team, and it is different according to the primary tumor location and tumor staging. The role of adjuvant radiotherapy remains controversial in high-grade EESS and due to the rarity of these cases there are limited data concerning the efficacy of adjuvant EBRT available from prospective randomized control clinical trials. 


\section{Introduction}

Endometrial stromal sarcoma (ESS) is a rare tumor, predominantly occurring as a primary tumor of the uterus. Rare cases of primary extrauterine ESS (EESS) have been reported. Low-grade ESS (LG-ESS) is more common than high-grade ESS (HG-ESS). Heterogeneous mixtures of morphologic and genetic features of LG-ESS and HG-ESS are distinct histopathological entities. The diagnosis is often made after hysterectomy (1).

ESS represents a rare diagnostic and a high- or low-grade tumor profile is distinguished by immunohistochemistry (IHC) tests (2). Up to $30 \%$ of patients have EESS at presentation. The treatment of ESS is multimodal, its management requiring a multidisciplinary team, and it is different according to the primary tumor location and tumor staging. The role of adjuvant radiotherapy remains controversial in high-grade EESS and due to the rarity of these cases there are limited data concerning the efficacy of adjuvant external radiotherapy (EBRT) available from prospective randomized control clinical trials (3). Here, we present five cases of ESS and one case of EESS. All cases received EBRT at the Radiotherapy Department of the Emergency Clinical Hospital 'Sfantul Apostol Andrei' Galati, during 2004-2020.

\section{Case reports}

Literature data support radiotherapy use for ESS with the aim of improving local tumor control, but without revealing increases in the survival rate with certainty $(1,4)$. We present five ESS cases and one EESS case. All cases received EBRT at the Radiotherapy Department of the 'Sfantul Apostol Andrei' Emergency Clinical Hospital of Galati, between 2004 and 2020, where we identified six EES cases. Five of them had ESS histology and were localized in the uterus [receiving treatment with EBRT in two-dimensional (2D) technique] and only one had ESS histology with extrauterine location [receiving palliative treatment with three dimensional conformational radiotherapy (3DCRT) technique, using the linear accelerator Elekta Synergy].

Clinical and therapeutic parameters of the EES and EESS patients are documented in Table I. The median age was 57.4 years.

Patients 1-5. Only one patient (patient no. 5) had IHC tests [HG-ESS that was estrogen receptor-negative (ER-), progesterone receptor-negative ( $\mathrm{PR}), \mathrm{CD} 10^{+}$, vimentin-positive (VIM+), Ki-67 30\%] because between 2004 and 2009, IHC assays were not performed routinely in our medical unit.

The surgical intervention, for patient nos. 1-5, consisted of a total hysterectomy with bilateral salpingo-oophorectomy. Patients who presented with post-surgical relapse risk factors such as vaginal or parameter invasion/extension received adjuvant radiotherapy.

Patient no. 2 presented with a second neoplastic disease occurring 9 years after the ESS diagnosis; this patient died in 2018 suffering from lung metastases from breast cancer; the breast cancer features were: pT2N0M0, invasive ductal carcinoma, ER=90\%, PR', Ki-67 >14\%. Taking into consideration the long survival and death of breast cancer complications, we consider that the histological aspects for this patient would have been LG-ESS.

Patient no. 3 presented for post-therapeutic controls, up until 2009, evading medical care since then. Patient no. 5 had a 23-month survival. We could not calculate a median survival due to the lack of data regarding all of the analyzed cases.

Patient no. 6. EESS was diagnosed in a 64-year-old patient (patient no. 6) with a personal history of hysterectomy and bilateral salpingo-oophorectomy in 1997, with the histopathological diagnosis of chronic cervicitis, uterine leiomyofibroma and uterine adenomyosis. In November 2019, the computer tomography (CT) scan examination revealed solid retroperitoneal lesions with heterogeneous contrast enhancement and areas of necrosis and calcification, localized in the left para-aortic region, inferior from the renal hilum with extension into the left psoas muscle $(58 / 66 \mathrm{~mm})$ and inferior pelvis, caudal from the common iliac artery bifurcation $(34 / 42 \mathrm{~mm}$ with $34 / 40 \mathrm{~mm}$ on the right and $22 / 33 \mathrm{~mm}$ with $44 / 62 \mathrm{~mm}$ on the left side). The retroperitoneal mass was biopsied, and the microscopic examination revealed a proliferation of monotonous ovoid to spindle cells, with scant cytoplasm, focally with 'clear cell' aspects, monomorphic nuclei and low mitotic activity, without any areas of necrosis.

Biomarker IHC studies and hematoxylin and eosin (H\&E) slide staining were necessary (5). Immunohistochemical staining revealed intense, diffuse positivity for ER isoform $\alpha$, diffuse positivity for CD10, focal actin and desmin positivity, and negativity for CD34, inhibin, calretinin, OCT3/4, PLAP, chromogranin A and Melan-A. The histopathological aspects of the mesenchymal tumor and the immunophenotype $\left(\mathrm{ER}^{+}, \mathrm{CD} 10^{+}\right)$revealed the diagnosis of LG-EESS (Figs. 1 and 2).

In January 2020, the magnetic resonance imaging (MRI) examination described a retroperitoneal heterogeneous tissue mass of 52/62 mm, with contrast enhancement and areas of necrosis, extending caudally from the left renal hilum having approximately $85 \mathrm{~mm}$ in length; the lesion encased the aorta and the left renal artery (which is still patent), it invaded the left renal vein and left psoas muscle, extending into the spinal canal (through the L2 left neural foramen in the anterior epidural space, extending cranially and caudally from L1 to L3). The mass also infiltrated the left sided para-vertebral muscles (Fig. 3).

The MRI from March 2020 revealed a solid expansive gadolinophilic retroperitoneal process, having axial measurements of 100/75 $\mathrm{mm}$ and invading the left psoas muscle, L1, L2 and with extension in the spinal canal (with extradural location), expanding across approximately $150 \mathrm{~mm}$, in between D1 and L4. The mass determined foraminal and spinal canal stenosis, with compressive effects on the horsetail roots (Fig. 4).

As treatment, the patient started hormone therapy with letrozol, and the oncology team decided to perform EBRT as palliative treatment at LINAC in 3DCRT technique, $\mathrm{TD}=30 \mathrm{~Gy} / 10 \mathrm{fr}, \mathrm{D} / \mathrm{fr}=300$ cGy on primary tumor and extension, with good treatment tolerance. 
Table I. Clinical and therapeutical parameters of the ESS and EESS patients.

\begin{tabular}{|c|c|c|c|c|c|c|c|c|}
\hline Patient no. & $\begin{array}{c}\text { Age } \\
\text { (years) }\end{array}$ & Year & $\begin{array}{c}\text { Surgical } \\
\text { intervention }\end{array}$ & HP & IHC & Risk factors & $\begin{array}{l}\text { Treatment intent } \\
\text { and total dose }\end{array}$ & $\begin{array}{l}\text { Survival } \\
\text { (months) }\end{array}$ \\
\hline 1 & 55 & 2004 & $\mathrm{TH}+\mathrm{BSO}$ & ESS G3 & - & Vagina & $\begin{array}{l}\text { Adjuvant EBRT } \\
\mathrm{TD}=50 \mathrm{~Gy}\end{array}$ & \\
\hline 2 & 43 & 2004 & $\mathrm{TH}+\mathrm{BSO}$ & ESS & - & Vagina & $\begin{array}{l}\text { Adjuvant EBRT } \\
\mathrm{TD}=50 \mathrm{~Gy}\end{array}$ & 168 \\
\hline 3 & 68 & 2006 & $\mathrm{TH}+\mathrm{BSO}$ & ESS & - & $\begin{array}{c}\text { Vagina }+ \\
\text { parameters }\end{array}$ & $\begin{array}{l}\text { Adjuvant EBRT } \\
\mathrm{TD}=50 \mathrm{~Gy}\end{array}$ & \\
\hline 4 & 55 & 2009 & $\mathrm{TH}+\mathrm{BSO}$ & ESS G3 & - & $\begin{array}{l}\text { Parameter, } \\
\text { miometer }\end{array}$ & $\begin{array}{l}\text { Adjuvant EBRT } \\
\mathrm{TD}=50 \mathrm{~Gy}\end{array}$ & \\
\hline 5 & 66 & 2016 & $\mathrm{TH}+\mathrm{BSO}$ & ESS & $\begin{array}{c}\text { HG-ESS; } \\
\text { ER-, PR CD } 10^{+}, \\
\text {VIM }^{+}, \text {Ki-67 30\% }\end{array}$ & & $\begin{array}{l}\text { Adjuvant EBRT } \\
\mathrm{TD}=50 \mathrm{~Gy}\end{array}$ & 23 \\
\hline 6 & 64 & 2020 & & EESS & & & $\begin{array}{l}\text { Palliative EBRT } \\
\mathrm{TD}=30 \mathrm{~Gy}\end{array}$ & 11 \\
\hline
\end{tabular}

HP, histopathology; IHC, immunohistochemistry; TH+BSO, total hysterectomy + bilateral salpingo-oophorectomy; EBRT, external beam radiotherapy; ESS, endometrial stromal sarcoma; EESS, extrauterine endometrial stromal sarcoma; HG-ESS, high-grade endometrial stromal sarcoma.
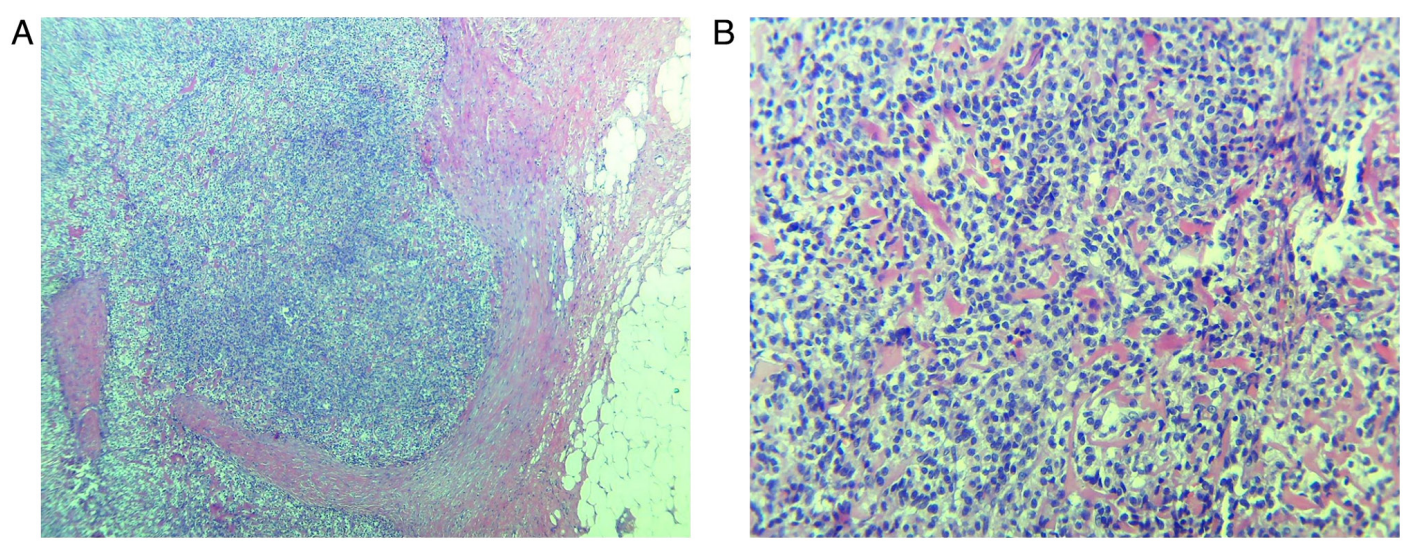

Figure 1. Histopathological aspects of low-grade EESS. (A) Proliferation of monotonous ovoid to spindle tumor cells, involving the adipose tissue. H\&E stain; magnification, x40. (B) Tumor cells are uniformly small, with oval-round nuclei and they focally whorl around small vessels. H\&E stain, magnification, x200. EESS, extrauterine endometrial stromal sarcoma; H\&E, hematoxylin and eosin.

\section{Discussion}

Most of the information concerning uterine sarcomas available in the medical literature is based on small series or case reports, which have analyzed the effects of adjuvant radiotherapy on the main histological subtypes. When grouping all of the histological subtypes, most studies have shown that adjuvant radiotherapy reduces the local and regional recurrence rates without having an overall survival advantage $(4,6)$. Endometrial stromal sarcoma (ESS) patients receiving adjuvant radiotherapy have registered improved local control, as compared to patients undergoing surgical treatment alone (4).

Wang et al (7) retrospectively reviewed 152 patients with stage I to II resected low-grade (LG)-ESS; the patients were included and analyzed for 20 years (1998-2018). Forty patients received adjuvant radiotherapy (EBRT group) and 112 patients did not receive adjuvant radiotherapy (no EBRT group). For all patients in the EBRT group, adjuvant radiotherapy significantly improved the disease-free survival (DFS) $(\mathrm{P}=0.008)$ and pelvic failure-free survival ( $\mathrm{PFFS})(\mathrm{P}=0.004)$. This study indicated that adjuvant radiotherapy significantly improved DFS and PFFS with tolerable adverse effects, especially in patients with stage IB to IIB disease. Despite several limitations (unequal number of patients in the two groups; retrospective single-center study), this is the largest population-based study exploring adjuvant radiotherapy in resected, early-stage LG-ESS patients and could be a valuable reference that provides guidance for radiotherapy treatment selection in specific subgroups (7).

A retrospective analysis was conducted in 2009 and it included 3,650 patients with uterine sarcoma; it was conducted using the National Oncology Database and had a median 

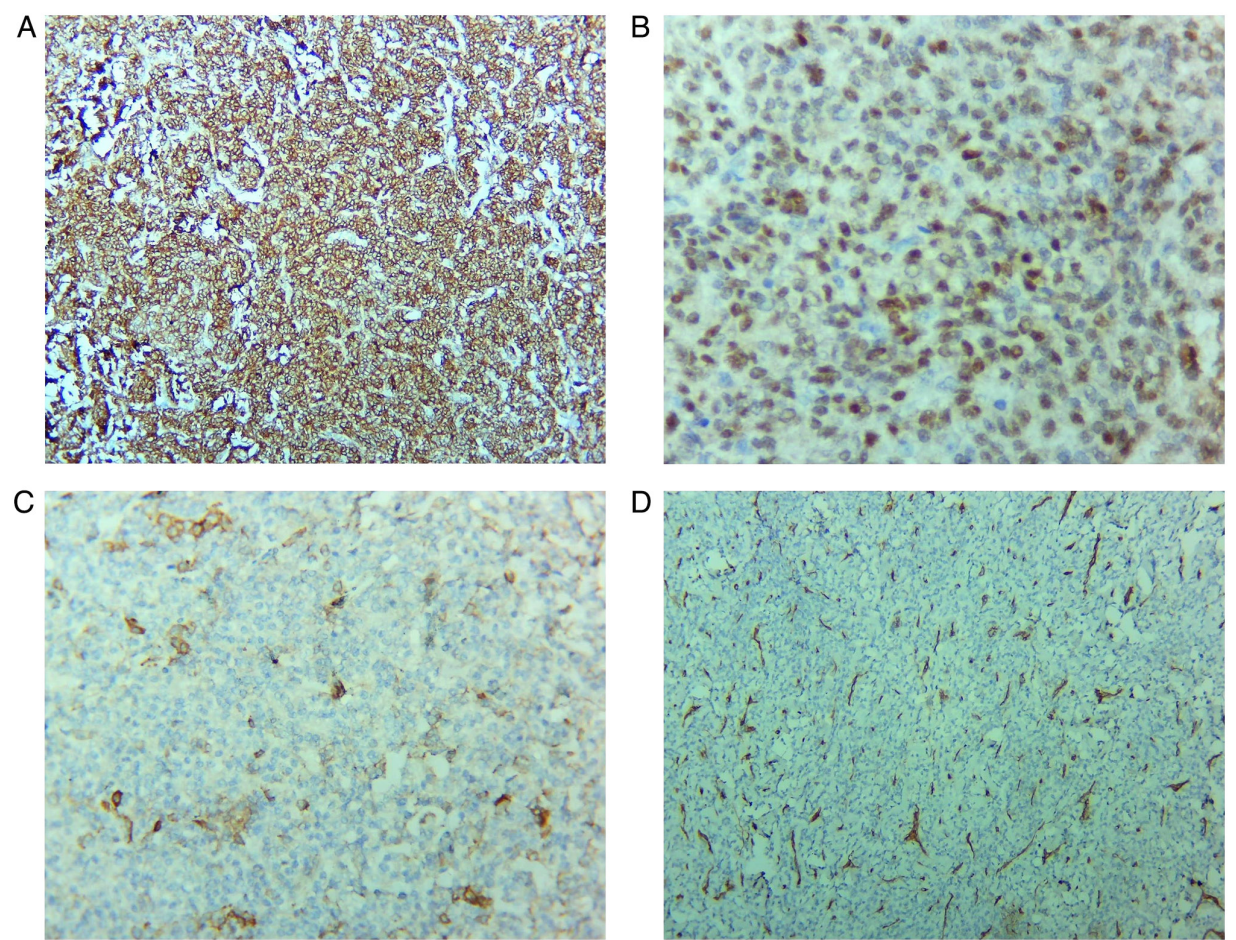

Figure 2. Immunohistochemistry staining. (A) Intense, diffuse positivity for CD10; magnification, x100. (B) Tumor cells show nuclear positivity for ER, with variable intensity; magnification, x400. (C) Focal positivity for actin (clone HHF35); magnification, x200. (D) CD34 is negative in the tumor cells and positive in the blood vessels network; magnification, x100. ER, estrogen receptor. HHF35, anti-muscle actin antibody.
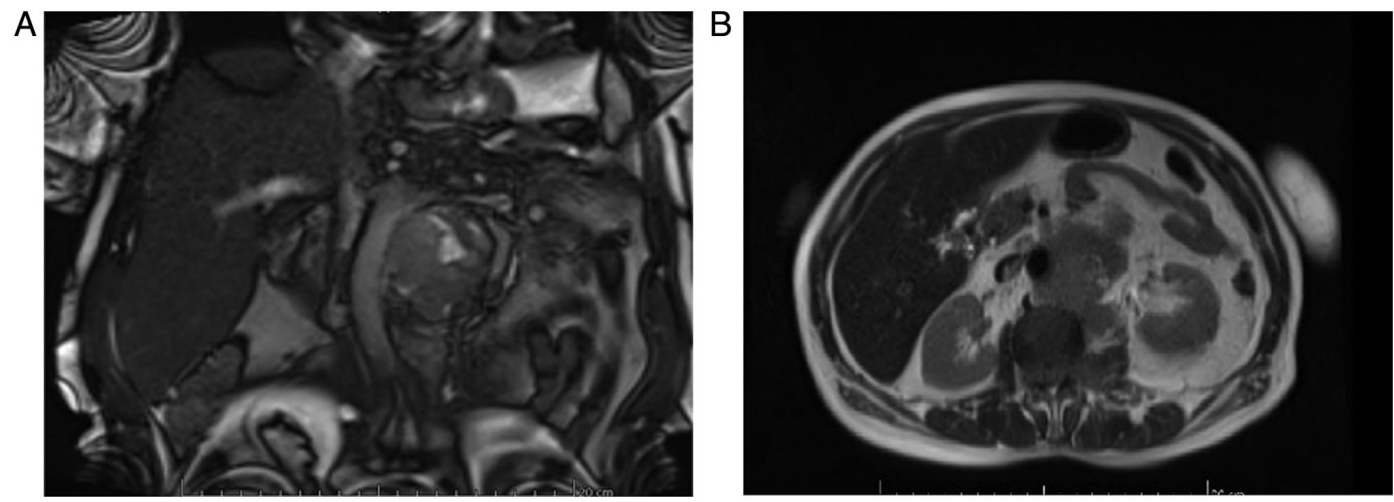

Figure 3. (A and B) Patient no. 6. January 2020 MRI examination; retroperitoneal heterogeneous tissue mass. MRI, magnetic resonance imaging.
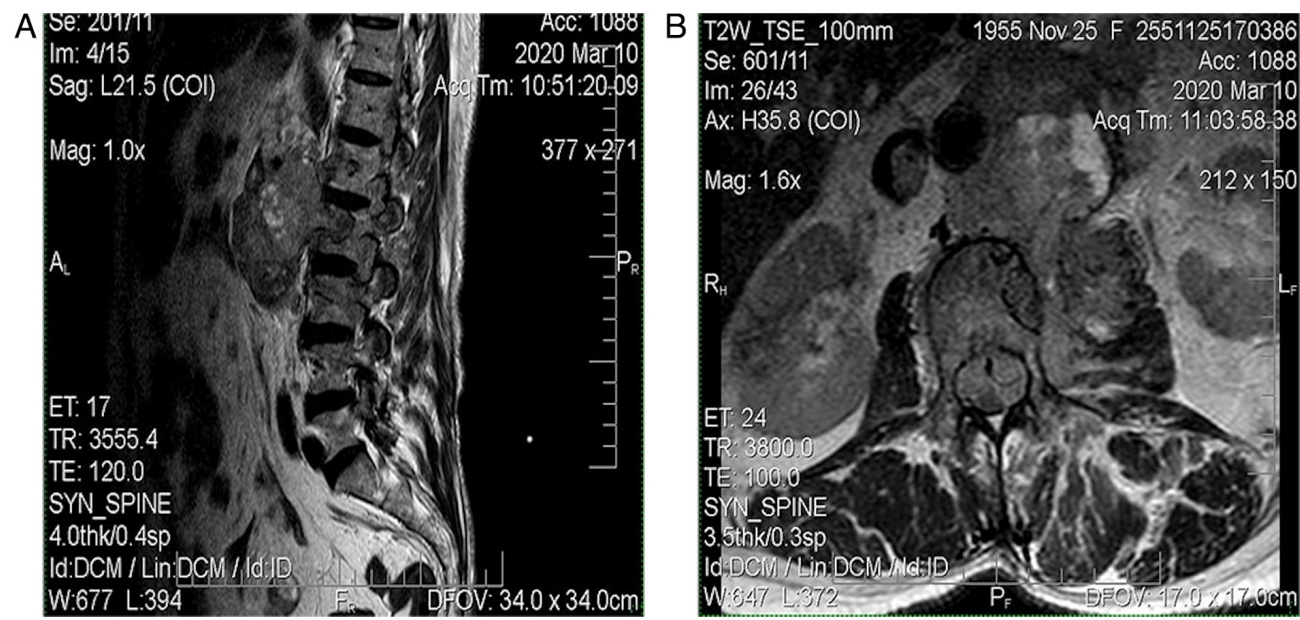

Figure 4. (A and B) Patient no. 6. March 2020 MRI; solid expansive gadolinophilic retroperitoneal process. MRI, magnetic resonance imaging. 
follow-up period of 59 months, with a 5-year overall survival of $37 \%$. Use of adjuvant radiotherapy was not predictive for overall survival. For non-metastatic cancer patients receiving definitive surgery, the 5-year local-regional failure-free survival (LRFFS) was $87 \%$ (8).

ESS is a rare diagnosis, usually presenting as a leiomyoma of the uterus. The symptoms are nonspecific, patients complaining most frequently of abnormal uterine bleeding. An early diagnosis is essential because patient survival is directly related to the tumor stage. Uterine sarcomas most often affect postmenopausal women (9).

The gross appearance of LG-ESS is characterized by several confluent tumor masses having a tan to yellow cut surface, sometimes presenting tumor plugs with worm-like appearance which permeate the myometrium or which can be found in blood vessels. The extrauterine spread of LG-ESS is indicated by some palpable tumor cords in the parauterine tissues. In contrast, high grade (HG)-ESS presents as large tumor masses (up to $9 \mathrm{~cm}$ in diameter) with extensive permeation of the myometrium; in some cases, the extrauterine spread can be more extensive than the uterine tumor itself (10-13).

The microscopic features of LG-ESS are characterized by an endometrial stromal nodule-like cytology (small cells, uniform round-oval nuclei, scant cytoplasm) with tongue-like/island-like invasion of the myometrium, sometimes also with lymphovascular invasion. It can have $<5$ mitoses/10 high-power fields (HPF), with limited, ischemic-type areas of necrosis and also various types of differentiation: smooth or skeletal muscle, adipose, sex cord or even glandular elements $(10-12,14)$.

HG-ESS can develop from a LG-ESS, and it presents with high-grade round cells ('small, round, blue-cell tumor'-like appearance), having scant-to-moderate cytoplasm and round and slightly irregular nuclei (larger than those of LG-ESS, approximately 4-6 lymphocytes) which sometimes can be pleomorphic; some also have low-grade areas with spindle-cell appearance, or with sex cord differentiation. The tumor cell growth pattern is frequently characterized by tight nests, or it can be rosette-like, pseudopapillary or pseudoglandular. The tumor has a fine network of vessels which are curvilinear or arborizing. The mitotic activity exceeds that of 10/10 HPF and the tumor mass has frequent areas of necrosis and lymphovascular invasion. The low-grade areas with bland spindle-cells can have various morphological aspects ranging from fibroblastic to fibrous to fibro-myxoid, with a low number of mitoses (a maximum of 3 mitoses/10 HPF) and no necrosis (typically). HG-ESS metastases can have either round, spindle-cell or round and spindle-cell morphology (10-12).

In general, the IHC profile of LG-ESS is characterized by CD10, ER and PR positivity, with possible positive markers: keratin, calretinin, and actin. These tumors can also be positive for other markers, according to the type of cell differentiation found (desmin, caldesmon positivity in smooth muscle differentiated cells, calretinin, inhibin, WT1, CD99, Melan-A in sex cord differentiated areas) (10-12,15).

HG-ESS has a rather similar IHC profile, characterizing each of the cellular components; thus, the spindle cells are positive for CD10, ER, PR, while the round cells are negative for these markers, but are highly positive for cyclin-D1.
Markers such as caldesmon, desmin, SMA, EMA, Melan-A or pankeratin are negative $(10-12,15,16)$.

Differential diagnosis for uterine LG-ESS includes cellular leiomyoma, intravascular leiomyomatosis, leiomyosarcoma, HG-ESS, gastrointestinal stromal tumor (GIST), perivascular epithelioid cell tumor (PEComa), gland-poor adenomyosis. Proper diagnosis requires attention to histological aspects and the use of an adequate panel of IHC markers $(10-12,16)$. Vroobel et al framed an immunopanel for the differential diagnosis of extrauterine neoplasms, which can be helpful in distinguishing LG-ESS from the entities mentioned above, but also from granulosa cell tumor, desmoplastic small round cell tumor or even lymphoma (12). HG-ESS needs to be differentiated from epithelioid leiomyosarcoma, PEComa of gynecological origin, epithelioid GIST with pelvic location, or undifferentiated uterine sarcoma (UUS), mainly based on histomorphology and immunophenotype (17).

In the specialty literature, the ESS relapse pattern is limited. Zhou et al (18) demonstrated that relapse of LG-ESS was $12.3 \%$ (14 of 114 included patients) mainly due to distant metastasis $(64.3 \%, 9 / 14)$ and only $5 / 14$ were pelvic recurrences. The median time of recurrences was 50 months (range, 6-169 months). In this study, all patients performed hysterectomy, ovarian preservation was performed in 20/114 of cases, adjuvant radiotherapy was performed in $31.6 \%$ of cases, $49.1 \%(56 / 114)$ patients received postoperative chemotherapy (median 3 courses) and $9.6 \%$ of patients received endocrine therapy. None of the analyzed factors, such as ovarian preservation, chemotherapy, adjuvant radiotherapy and endocrine therapy, did not influence DFS. The benefit of EBRT was not evidenced in this study.

Up to $30 \%$ of women with LG-ESS have an extrauterine disease at presentation. Preoperative diagnosis is often difficult and approximately $75 \%$ are diagnosed as benign leiomyoma. Endometrial curettage and histopathological examination (HPE) do not help due to the similarity with normal endometrium (2). A case of ESS was reported in a 30-year-old woman with ultrasound and Doppler findings suggestive of uterine leiomyoma. Sarcomatous change was suspected in view of the rapid enlargement of the tumor. Endometrial aspiration revealed a secretory endometrium with neoplastic cells and thus a hysterectomy was performed (19). Another rare presentation of LG-ESS was a low-grade endometrial sarcoma of the endocervix, clinically presenting as a soft hemorrhagic mass in the posterior cervix, appearing as a degenerated leiomyoma (20). Some recent data on the low numbers of patients with LG-ESS appear to show an incidence of nodal involvement higher than previously expected, thus suggesting a purpose for lymphadenectomy in this malignancy (21). Hormone therapy with gonadotropin releasing hormone $(\mathrm{GnRH})$ analogues and aromatase inhibitors are suggested for LG-ESS stage 3-4 and for recurrent disease $(22,23)$.

In a study by Chu and colleague, $75 \%$ of patients with stage I disease did not recur when treated with adjuvant medroxyprogesterone acetate compared with $29 \%$ similar stage patients who did not receive treatment (24).

Recommended adjuvant therapy options for stage I ESS include observation (especially in the case of menopause or prior bilateral salpingo-oophorectomy) or estrogen blockade. Postoperative estrogen blockade is recommended for stages II 
to IV ESS. Adjuvant EBRT may be added for stage II-IVA. Hormone therapy includes aromatase inhibitors, megestrol acetate, or medroxyprogesterone acetate (25).

Case series of patients with ESS suggest long disease-free intervals in the absence of specific therapy and raise questions about the use of adjuvant EBRT that has been demonstrated to reduce local recurrence rates but with limited effect on survival $(6,26,27)$.

Regarding HG-ESS, the role of adjuvant EBRT in non-metastatic disease is controversial (3).

The treatment is multimodal, with a management requiring a multidisciplinary team, and a difference regarding the primary tumor location and staging of the tumor. Rarely ESS is initially present in an extrauterine site. IHC will help in the detection of the specific tumor markers. In our cases, the IHC of LG-ESS and HG-ESS revealed a completely different biological aggressiveness and clinical behavior. Management was different regarding the primary tumor location and staging of the tumor. Initial surgical approach is considered as the optimal treatment, but the aim of post-operative radiation treatment remains uncertain. EBRT may have broader indications than what is currently accepted in clinical practice. The purpose of adjuvant EBRT in non-metastatic disease is controversial.

\section{Acknowledgements}

Not applicable.

\section{Funding}

No funding was received.

\section{Availability of data and materials}

The information generated and analyzed in regards to the case reports is available from the corresponding author on reasonable request.

\section{Authors' contributions}

LFR, DF, RMA, LG, AMI, MED, MC, EN, ALT, MSC, ML and AIN contributed to the acquisition, analysis and interpretation of the data; they made substantial contributions to the conception and design of the work, and supervised and substantially revised this work. All authors had equal contributions, equal participation and the same rights to this article. All authors have read and agreed to the published version of the manuscript.

\section{Ethics approval and consent to participate}

The Ethics Committee of 'Sfantul Apostol Andrei' Emergency Clinical Hospital of Galati has granted approval for this study with the decision number 8540 from 09.04.2021.

\section{Patient consent for publication}

Consent for participation was granted by all of the patients and is part of the personal observation sheet.

\section{Competing interests}

The authors declare that they have no competing interests.

\section{References}

1. Sampath S and Gaffney DK: Role of radiotherapy treatment of uterine sarcoma. Best Pract Res Clin Obstet Gynaecol 25: 761-772, 2011.

2. Jin Y, Pan L, Wang X, Dai Z, Huang H, Guo L, Shen K and Lian L: Clinical characteristics of endometrial stromal sarcoma from an academic medical hospital in China. Int $\mathbf{J}$ Gynecol Cancer 20: 1535-1539, 2010.

3. Reed NS, Mangioni C, Malmström H, Scarfone G, Poveda A, Pecorelli S, Tateo S, Franchi M, Jobsen JJ, Coens C, et al: Phase III randomised study to evaluate the role of adjuvant pelvic radiotherapy in the treatment of uterine sarcomas stages I and II: An European organisation for research and treatment of cancer gynaecological cancer group study (protocol 55874). Eur J Cancer 44: 808-818, 2008.

4. Valduvieco I, Rovirosa A, Colomo L, De San Juan A, Pahisa $J$ and Biete A: Endometrial stromal sarcoma. Is there a place for radiotherapy? Clin Transl Oncol 12: 226-230, 2010.

5. Bacinschi XE, Zgura A, Safta I and Anghel R: Biomolecular factors represented by Bcl-2, p53, and tumor-infiltrating lymphocytes predict response for adjuvant anthracycline chemotherapy in patients with early triple-negative breast cancer. Cancer Manag Res 12: 11965-11971, 2020.

6. Weitmann HD, Knocke TH, Kucera H and Pötter R: Radiation therapy in the treatment of endometrial stromal sarcoma. Int $\mathrm{J}$ Radiat Oncol Biol Phys 49: 739-748, 2001.

7. Wang W, Sun S, Miao Z, Hou X, Zhang F and Hu K: Adjuvant radiotherapy improved survival in stage I to II low-grade endometrial stromal sarcoma: A retrospective study of 152 cases. Front Oncol 10: 608152, 2021.

8. Sampath S, Schultheiss TE, Ryu JK and Wong JY: The role of adjuvant radiation in uterine sarcomas. Int $\mathbf{J}$ Radiat Oncol Biol Phys 76: 728-734, 2010.

9. Jain R, Batra S, Ahmad A, Elahi AA, Gupta M and Saith P: Low grade endometrial stromal sarcoma: A case report. Iran J Med Sci 40: 81-84, 2015.

10. Hoang L, Chiang S and Lee CH: Endometrial stromal sarcomas and related neoplasms: New developments and diagnostic considerations. Pathology 50: 162-177, 2018.

11. Lipsich F, Causa Andrieu PI, Wernicke A, Patrono MG, Napoli MN, Chacon CRB and Nicola R: Extra-uterine endometrial stromal sarcoma arising from deep infiltrating endometriosis. Clin Imaging 67: 250-254, 2020.

12. Vroobel KM, Karawita TS and Nafisa Wilkinson: New developments in endometrial stromal sarcoma. Diagn Histopathol 23: 311-322, 2017.

13. Ashraf-Ganjoei T, Behtash N, Shariat M and Mosavi A: Low grade endometrial stromal sarcoma of uterine corpus, a clinico-pathological and survey study in 14 cases. World J Surg Oncol 4: 50, 2006.

14. Seagle BL, Shilpi A, Buchanan S, Goodman C and Shahabi S: Low-grade and high-grade endometrial stromal sarcoma: A national cancer database study. Gynecol Oncol 146: 254-262, 2017.

15. Farhood AI and Abrams J: Immunohistochemistry of endometrial stromal sarcoma. Hum Pathol 22: 224-230, 1991.

16. Zhu XQ, Shi YF, Cheng XD, Zhao CL and Wu YZ: Immunohistochemical markers in differential diagnosis of endometrial stromal sarcoma and cellular leiomyoma. Gynecol Oncol 92: 71-79, 2004.

17. Ali RH and Rouzbahman M: Endometrial stromal tumours revisited: An update based on the 2014 WHO classification. J Clin Pathol 68: 325-332, 2015.

18. Zhou J, Zheng H, Wu SG, He ZY, Li FY, Su GQ and Sun JY: Influence of different treatment modalities on survival of patients with low-grade endometrial stromal sarcoma: A retrospective cohort study. Int J Surg 23: 147-151, 2015.

19. Puliyath G, Nair VR and Singh S: Endometrial stromal sarcoma. Indian J Med Paediatr Oncol 31: 21-23, 2010.

20. Hasiakos D, Papakonstantinou K, Kondi-Paphiti A and Fotiou S: Low-grade endometrial stromal sarcoma of the endocervix. Report of a case and review of the literature. Eur J Gynaecol Oncol 28: 483-486, 2007. 
21. Gadducci A, Cosio S, Romanini A and Genazzani AR: The management of patients with uterine sarcoma: A debated clinical challenge. Crit Rev Oncol Hematol 65: 129-142, 2008.

22. Palombaa S, Falboa A, Mocciaroa R, Russob T and Zulloa F: Laparoscopic treatment for endometrial cancer: A meta-analysis of randomized controlled trials (RCTs). Gynecol Oncol 112: 415-421, 2009.

23. Lindner T, Pink D, Kretzschmar A, Mrozek A, Thuss-Patience PC and Reichardt P: Hormonal treatment of endometrial stromal sarcoma: A possible indication for aromatase inhibitors. J Clin Oncol 23 (Suppl 16): S9057, 2005.

24. Chu MC, Mor G, Lim C, Zheng W, Parkash V and Schwartz PE: Low grade endometrial stromal sarcoma: Hormonal aspects. Gynaecol Oncol 90: 170-176, 2003.
25. Amant F, Coosemans A, Debiec-Rychter M, Timmerman D and Vergote I: Clinical management of uterine sarcomas. Lancet Oncol 10: 1188-1198, 2009.

26. Mansi JL, Ramachandra S, Wiltshaw E and Fisher C: Endometrial stromal sarcomas. Gynecol Oncol 36: 113-118, 1990.

27. Berchuck A, Rubin SC, Hoskins WJ, Saigo PE, Pierce VK and Lewis JL Jr: Treatment of endometrial stromal tumors. Gynecol Oncol 36: 60-65, 1990.

(7)(2) This work is licensed under a Creative Commons Attribution-NonCommercial-NoDerivatives 4.0 International (CC BY-NC-ND 4.0) License. 